NASA/CR-2004-213224

\title{
Progress in NEXT Ion Optics Modeling
}

Jerold W. Emhoff and Iain D. Boyd

University of Michigan, Ann Arbor, Michigan 
Since its founding, NASA has been dedicated to the advancement of aeronautics and space science. The NASA Scientific and Technical Information (STI) Program Office plays a key part in helping NASA maintain this important role.

The NASA STI Program Office is operated by Langley Research Center, the Lead Center for NASA's scientific and technical information. The NASA STI Program Office provides access to the NASA STI Database, the largest collection of aeronautical and space science STI in the world. The Program Office is also NASA's institutional mechanism for disseminating the results of its research and development activities. These results are published by NASA in the NASA STI Report Series, which includes the following report types:

- $\quad$ TECHNICAL PUBLICATION. Reports of completed research or a major significant phase of research that present the results of NASA programs and include extensive data or theoretical analysis. Includes compilations of significant scientific and technical data and information deemed to be of continuing reference value. NASA's counterpart of peerreviewed formal professional papers but has less stringent limitations on manuscript length and extent of graphic presentations.

- TECHNICAL MEMORANDUM. Scientific and technical findings that are preliminary or of specialized interest, e.g., quick release reports, working papers, and bibliographies that contain minimal annotation. Does not contain extensive analysis.

- CONTRACTOR REPORT. Scientific and technical findings by NASA-sponsored contractors and grantees.
- CONFERENCE PUBLICATION. Collected papers from scientific and technical conferences, symposia, seminars, or other meetings sponsored or cosponsored by NASA.

- SPECIAL PUBLICATION. Scientific, technical, or historical information from NASA programs, projects, and missions, often concerned with subjects having substantial public interest.

- TECHNICAL TRANSLATION. Englishlanguage translations of foreign scientific and technical material pertinent to NASA's mission.

Specialized services that complement the STI Program Office's diverse offerings include creating custom thesauri, building customized databases, organizing and publishing research results ... even providing videos.

For more information about the NASA STI Program Office, see the following:

- Access the NASA STI Program Home Page at http://www.sti.nasa.gov

- E-mail your question via the Internet to help@sti.nasa.gov

- Fax your question to the NASA Access Help Desk at 301-621-0134

- Telephone the NASA Access Help Desk at 301-621-0390

- Write to:

NASA Access Help Desk

NASA Center for AeroSpace Information 7121 Standard Drive

Hanover, MD 21076 
NASA/CR-2004-213224

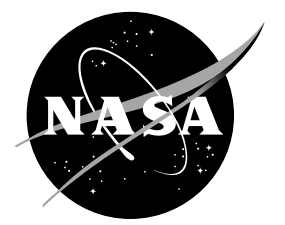

\section{Progress in NEXT Ion Optics Modeling}

Jerold W. Emhoff and Iain D. Boyd

University of Michigan, Ann Arbor, Michigan

Prepared for the

40th Joint Propulsion Conference and Exhibit

cosponsored by the AIAA, ASME, SAE, and ASEE

Fort Lauderdale, Florida, July 11-14, 2004

Prepared under Grant NAG3-2497

National Aeronautics and

Space Administration

Glenn Research Center 


\title{
Acknowledgments
}

\begin{abstract}
Support for this work is provided in part by NASA Glenn Research Center, Grant NAG3-2497, with George Soulas as the technical monitor. Support is also provided by a Michigan Space

Grant Consortium Graduate Student Fellowship.
\end{abstract}

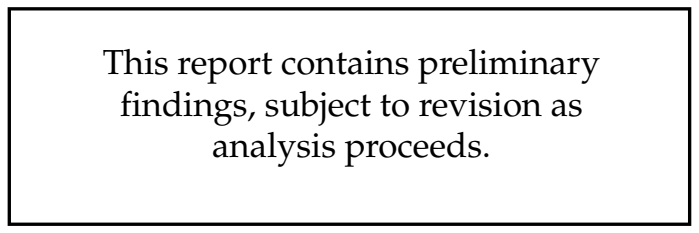

Available from

NASA Center for Aerospace Information 7121 Standard Drive

Hanover, MD 21076
National Technical Information Service 5285 Port Royal Road Springfield, VA 22100

Available electronically at http:/ /gltrs.grc.nasa.gov 


\title{
Progress in NEXT Ion Optics Modeling
}

\author{
Jerold W. Emhoff and Iain D. Boyd \\ University of Michigan \\ Ann Arbor, Michigan 48109
}

\begin{abstract}
Results are presented from an ion optics simulation code applied to the NEXT ion thruster geometry. The error in the potential field solver of the code is characterized, and methods and requirements for reducing this error are given. Results from a study on electron backstreaming using the improved field solver are given and shown to compare much better to experimental results than previous studies. Results are also presented on a study of the beamlet behavior in the outer radial apertures of the NEXT thruster. The low beamlet currents in this region allow over-focusing of the beam, causing direct impingement of ions on the accelerator grid aperture wall. Different possibilities for reducing this direct impingement are analyzed, with the conclusion that, of the methods studied, decreasing the screen grid aperture diameter eliminates direct impingement most effectively.
\end{abstract}

\section{Introduction}

NASA'S Evolutionary Xenon Thruster (NEXT) ${ }^{1}$ is currently under development to follow the success of the NSTAR ion thruster. ${ }^{2}$ NEXT has a $40 \mathrm{~cm}$ beam extraction diameter - giving more than twice the area of the $30 \mathrm{~cm}$ NSTAR thruster. This allows a larger amount of thrust, and a redesigned discharge chamber gives a flatter beam current density profile, which reduces erosion of the ion optics. ${ }^{3}$ The NEXT thruster also has an accelerator grid $50 \%$ thicker than the NSTAR thruster. This is to allow for a longer life, as the thicker grid can withstand more erosion on the downstream face, and also prevents electron backstreaming more effectively than a thinner grid.

Ion optics modeling is being used to aid the development of NEXT, especially in the area of erosion and life prediction. This area is the most difficult to address experimentally, as it requires operating the thruster for a very long time in order to determine the erosion behavior and life-limiting mechanisms. In this paper the ability of the model to simulate optics erosion is improved and applied to a specific situation. The accuracy of the potential field solver used in the ion optics model is analyzed and improved, with results showing improvement compared to experimental data. The model is also applied to simulate erosion in the apertures at the outer edge of the extraction grids. In these apertures, extremely low beamlet currents cause crossover of the beam ions and direct impingement on the accelerator grid aperture wall. Methods for mitigating this erosion are explored.

\section{Model Operation}

The computational model simulates a single 2-D axisymmetric aperture in an ion thruster. A computational mesh composed of evenly spaced rectangular cells is used to track particles in the simulation. The optics of the thruster are simulated using boundary cells in the domain. These cells may be arranged in an irregular way, allowing the simulation of cusps on the barrels of the grids. A typical computational domain is shown in Figure 1.

The code uses the Particle-In-Cell ${ }^{4}$ (PIC) method to simulate xenon ions, xenon neutrals, and doubly charged xenon ions. Each computational particle has a numerical weight that indicates the actual number of atoms represented by the particle. Flow field quantities for each cell are obtained by averaging the properties of all the particles in the cell, taking into account the weight of each particle. The potential field accelerates ions selfconsistently and electrons are modeled as a fluid. The potential solver is described in further detail below. The direct simulation Monte Carlo (DSMC) method ${ }^{5}$ is used for processing particle collisions. Both charge exchange (CEX) and momentum exchange collision types are simulated.

For the simulations performed in this paper, neutrals are not included in order to increase the speed of the model. In the potential mesh refinement study, the model is modified to allow a finer potential mesh without refining the particle mesh. However, cases at a refined particle mesh are also simulated in order to verify the potential mesh refinement. Figure 2 shows both potential mesh refinement and particle mesh refinement. If the entire box shown in the figure is considered the nominal cell size, then the particle cells are refined by two times, and the potential mesh is refined by another four times, giving a total refinement level of 8x. For the potential mesh refinement study, collisions are not processed. 
In each simulation, the potential field is first initialized using only the boundary conditions, then ions are injected. On the order of 60,000 iterations are performed to reach a steady-state of ion flow, after which data is sampled for approximately 10,000 iterations. The total time for this process depends on the domain size and the refinement level. For the coarsest mesh, such a case takes less than 15 minutes on a $3.06 \mathrm{GHz}$ Pentium 4 machine. For a mesh using potential cells that are refined by a factor of eight in each direction, the same case takes over 8 hours.

\section{Field Solver Error Analysis}

The cells used in the simulation are rectangular and constant in size throughout the domain. To find the potential field, charged particles have their weight distributed to the four mesh nodes surrounding the particle's position. The electron density at each node is determined by the Boltzmann relation, shown in Equation 1.

$$
n_{e}=n_{e, 0} \exp \left[\frac{\phi-\phi_{0}}{T_{e, 0}}\right]
$$

Here, $n_{e}$ is the electron density in $\mathrm{m}^{-3}, n_{e, 0}$ is the reference electron density in $\mathrm{m}^{-3}, \phi$ is the potential at the node in Volts, $\phi_{0}$ is the reference potential in Volts, and $T_{e, 0}$ is the reference electron temperature in $\mathrm{eV}$. Two separate reference states are used in the model - an upstream state and a downstream state. The upstream reference density is the discharge chamber ion density, the reference potential is the discharge chamber plasma potential, and the reference electron temperature is the discharge chamber electron temperature. The downstream reference density is the average ion density at the downstream boundary of the computational domain. The downstream reference potential is the beam plasma potential relative to ground, and the downstream reference electron temperature is the beam plasma electron temperature. The two reference states are divided by the inter-grid region, where no electrons exist. The exception to this is for conditions near or past the electron backstreaming limit, where electrons are able to flow upstream into the discharge chamber from the downstream plasma. In this case, the boundary between the two electron populations is maintained at the axial location of the downstream face of the accelerator grid.

Given the ion and electron densities at each node, and the boundary conditions, Poisson's equation is solved.

$$
\nabla \phi=\frac{e}{\varepsilon_{0}}\left(n_{e}-n_{i}\right)
$$

In this case, an alternating-direction-implicit (ADI) solver is used. At each iteration of the solver, the amount of change in the potential field, the residual, is reduced. The potential is updated based on this reduction in residual, the electron densities are recalculated, and the next iteration begins. This process is continued either until the residual is below a threshold value or for a set number of iterations.

An initial study compared the simulation's prediction of the electron backstreaming limit to experimental results. In this study, the model predicted that an accelerator grid voltage of $-140 \mathrm{~V}$ was required in order for backstreaming to occur; while experimental data indicates that backstreaming occurs at about $-170 \mathrm{~V}$ (Ref. 3). An error analysis study for the potential solver is undertaken as a possible cause of this difference.

Two types of error are present in the potential solver. One type is error due to the residual, where the solution is not converged completely. Running the field solver for a larger number of iterations reduces this error. The mesh itself causes the other type of error, since coarser meshes give larger errors. In previous simulations, the mesh used by the potential solver was determined solely by the particle flow properties such as density and velocity. This led to a coarse mesh for solving the potential field, even though the mesh size was appropriate for the particle properties. Figure 3 demonstrates these two types of error. In this plot, the potential field for a case with the potential mesh refined by 8 times is subtracted from a case with the mesh refined by 16 times. The high difference in potential near the ion optics inner corners and towards the centerline of the accelerator grid aperture is caused by coarseness of the $8 \mathrm{x}$-refined potential mesh. The high gradient in potential between the optics, especially at the corners, causes this large error. The change in potential in the downstream region is due to residual error in the 16x-refined case. The regions immediately upstream and downstream of the ion optics are the most sensitive to residual error because these regions have the highest electron density gradients. The error observed in the electron backstreaming study was likely due to mesh coarseness rather than residual error, as the residual drops quickly for coarse meshes. 
A mesh refinement study is undertaken to determine the magnitude of the error in the potential field as a function of both the mesh size and the residual. As noted above, the model is modified to allow refinement of the potential field mesh without refining the particle cell size. This allows more accurate potential field results without increasing the number of particles in the simulation. This method is verified by performing simulations with refined particle cells instead of or in addition to potential mesh refinement. The domain geometry used for the refinement study is similar to the NEXT geometry, with all dimensions rounded to exact numbers in order to allow better accuracy when dividing cells for refinement. Cusps are not simulated in this study except where noted.

Figure 4 shows the maximum difference in the potential field as a function of the residual, for several different mesh refinement levels. The difference in each case is compared to the lowest residual calculated for that refinement level, which is not shown on the plot. So, even though the potential difference for each data set goes to zero, the total error is still finite due to mesh coarseness error. For these cases, a sample simulation at the $4 x$ level of particle cell refinement gives the ion density used in the potential solver. This allows the potential to be computed consistently across the refinement levels by interpolating the densities appropriately. Using a constant density field also allows a shorter simulation to be performed, as no particles need to be injected, initialized, or sampled. The potential field is simply solved based on the ion density field, requiring no other operation of the code.

As the plot shows, the error drops linearly with the residual. As the refinement level increases, the residual must drop below a lower level to reach the same difference, by a factor of about $10^{-1 / 2}$. This means that the computational time required as the mesh is refined increases greatly, because the time required for a given number of iterations increases by a factor of four every time the mesh is refined by a factor of two. Since the residual must be lower as well, approximately 3 times as many iterations are required, increasing the computational cost by a factor of 12 for every level of refinement.

The maximum difference in potential as the mesh is refined is plotted in Figure 5. In these cases, the different lines correspond to different levels of particle cell refinement, and that refinement level multiplied by the potential mesh refinement gives the total refinement level. For example, a case with two-times refined particle cells and a $2 x$ refined potential mesh has a $4 x$ total refinement relative to the nominal cell size. Full simulations with moving ions give the results seen in the figure, except for the constant density case. The data from the constant density case comes from the data shown in Figure 4, using the points of lowest residual for each mesh refinement level. All data sets are compared against the 16 times refined case with $4 \mathrm{x}$ refined particle cells.

The difference in potential drops dramatically as the total refinement level is increased. Except for the $2 \mathrm{x}$ particle cell, 16x total refinement case, the amount of particle cells does not matter. In the anomalous case, the potential solver does not properly converge with a small residual. The constant density results give the same answer as the full simulation cases, verifying the use of the constant density field for determining the error in the potential field.

The $8 \mathrm{x}$ refined case shows a difference in potential below $5 \mathrm{~V}$, which is sufficient for the purposes of the model when applied to ion thruster optics, allowing less computation than the 16x refined case. Computationally, it makes sense to keep the level of error the same for both types of error. So, if a $5 \mathrm{~V}$ error is expected due to coarseness of the mesh, then the residual error should not be reduced below 5V. Otherwise, computational time is wasted because no matter how low the residual error is, the error in the potential will still be $5 \mathrm{~V}$.

The inclusion of cusps does not affect the results of this study. Constant ion density field simulations with cusps give the same change in potential difference as the residual decreases, and also the same drop in potential difference as the mesh is refined.

The electron backstreaming study was performed again, this time with the potential mesh refined to the $8 \mathrm{x}$ level, in order to test the effectiveness of using the mesh refinement. The domain geometry used here is the same as the NEXT geometry, with simulation of cusps included. To determine the electron backstreaming limit, the accelerator grid potential is increased until the minimum centerline potential is above the potential of the downstream plasma, which is $22 \mathrm{~V}$. At this point, electrons are able to flow upstream into the discharge chamber.

Figure 6 plots the minimum centerline potential as a function of the accelerator grid potential, as well as the $22 \mathrm{~V}$ limit. Both $8 \mathrm{x}$ refined and unrefined data are shown. A $10 \mathrm{~V}$ difference is seen between the old results and the new, with the same slope of line. However, even the new results are below the predicted electron backstreaming limit of $-170 \mathrm{~V}$ by $20 \mathrm{~V}$. This may indicate a need for further refinement, or it could be caused by another source, such as the electron model used, or the boundary conditions in the domain.

\section{Outer Radial Aperture Erosion Study}

In the 2000 hour wear test of the NEXT ion thruster ${ }^{6}$, a large amount of erosion was observed in the apertures at the edge of the extraction grids compared to the central regions ${ }^{7}$. The erosion was in the form of irregular star 
patterns in general, and occurred past the $15.1 \mathrm{~cm}$ radius point on the grids. Several factors most likely caused the erosion: the current densities in this region were low compared to the rest of the thruster, the aperture diameters were smaller than nominal, and there was also some misalignment in the optics. Together, these resulted in severe crossover of beam ions and erosion due to direct impingement.

The nature of the erosion observed experimentally was largely three-dimensional in nature, so the 2-D axisymmetric model used here cannot reproduce the erosion behavior completely. However, it can predict direct impingement of beam ions on the accelerator grid aperture wall, even if it cannot give a pattern to the erosion. All cases performed in this study have a four-times potential mesh refinement level, for greater accuracy.

Figure 7 shows the erosion rate on the accelerator grid aperture wall as a function of beamlet current, with the grid geometry used in the NEXT wear test. As the beamlet current drops, the erosion rate increases dramatically, before reaching a maximum and dropping again. All erosion here is due to direct impingement, as no CEX collisions are processed in these simulations. The erosion rates do not match the rates seen in the wear test, but this is expected due to the three dimensional nature of the actual erosion. The correct trends are observed however, and direct impingement is predicted for the beamlet currents the outer apertures would process.

A method of eliminating this erosion is desired, since the large amounts of erosion caused abnormal amounts of molybdenum to be present in the system. This could cause contamination of the spacecraft, or failure of the ion thruster due to a grid short. One proposed method of reducing the erosion is to reduce the screen grid aperture diameter. This has the effect of reducing the beamlet diameter, and the amount of crossover. However, it decreases the ion transparency and thus the efficiency of the thruster. Figure 8 plots the effect of the screen grid aperture diameter on both the erosion rate and the beamlet current.

As the aperture diameter is decreased, the erosion rate due to direct impingement decreases linearly. The beamlet current also drops. However, the erosion rate drops by a factor of 6 or more for a decrease in screen grid aperture diameter of 0.015 in., while the drop in beamlet current for the same decrease is only $13 \%$. Given that the outer apertures only process a small amount of the total beam current, this slight drop in beamlet current will not greatly affect the total beam current.

Another possible method for reducing the erosion rate is by increasing the aperture diameter of the accelerator grid. This has the disadvantage of more easily allowing electron backstreaming, it weakens the structural strength of the accelerator grid, and also allows a more divergent beamlet. When the diameter is increased by 0.01 in. from nominal at a beamlet current of $7.2 \mu \mathrm{A}$, the erosion rate is reduced by $46 \%$. This is a significant drop in erosion rate, but it is still much smaller than the reduction seen when the screen grid aperture diameter is reduced by the same amount.

The effects of the grid gap and the discharge chamber electron temperature are also examined. Increasing the grid gap by $0.002 \mathrm{in}$. from nominal reduces erosion by $20 \%$ at a beamlet current of $13.8 \mu \mathrm{A}$, and reducing the grid gap by the same amount increases the erosion rate by $33 \%$. Changing the grid gap is not an effective way to eliminate direct impingement however, as it greatly affects the performance of the other parts of the thruster. The discharge chamber electron temperature's effect on the erosion rate is examined because lower energy electrons may be present at the outer edges of the discharge chamber. No large effect is seen, as a $83 \%$ drop in electron temperature only reduced the erosion rate by $22 \%$ at a beamlet current of $26.5 \mu \mathrm{A}$ and the wear test geometry.

The results above indicate that the best method for reducing the erosion due to direct impingement is by reducing the screen grid aperture diameter in the outer region. Although the screen grid ion transparency drops a small amount, the erosion rate is reduced dramatically. One method not explored here is complete masking of the outer apertures. This completely eliminates the possibility of direct impingement, and therefore may be a more efficient and desirable solution than reducing the screen grid aperture diameter.

\section{Conclusion}

The error in the potential field solver has been characterized. It occurs in two forms: a residual error and a mesh coarseness error. Running the field solver for a larger number of iterations reduces the residual error, and refining the potential solver mesh reduces the coarseness error. The particle mesh does not need to be refined in order to obtain more accurate potential field results, but even so, the computational time required increases by a factor of 12 with every level of refinement of the potential mesh. Using an $8 \mathrm{x}$ mesh refinement, results for electron backstreaming improved by $10 \mathrm{~V}$ over un-refined results, although a $20 \mathrm{~V}$ difference from experimental results remains.

Erosion due to direct impingement in the outer apertures of the NEXT thruster has been explored. The simulation was able to predict direct impingement of beam ions on the accelerator grid aperture wall at conditions seen in the NEXT 2000 hour wear test. Simulations were conducted in order to find an efficient and simple method 
for eliminating the erosion due to direct impingement. Of the methods studied, decreasing the screen grid aperture diameter was found to be the most effective for reducing direct impingement of ions, even though it caused a slight decrease in the beamlet current contribution from the outer apertures. Increasing the accelerator grid aperture diameter also decreased the erosion rate, but to a lesser extent. The effects of grid gap and discharge chamber electron temperature were also explored. It was found that increasing the grid gap resulted in a decreased erosion rate, as did decreasing the electron temperature, although neither decrease was significant.

\section{References}

${ }^{1}$ Patterson, M.J., Foster, J.E., Haag, T.W., Rawlin, V.K., Soulas, G.C., and Roman, R.F., “NEXT: NASA's Evolutionary Xenon Thruster," AIAA 2002-3832, July 2002.

${ }^{2}$ Polk, J.E., Brinza, D., Kakuda, R.Y., Brophy, J.R., Katz, I., Anderson, J.R., Rawlin, V.K., Patterson, M.J., Sovey, J., and Hamley, J., "Demonstration of the NSTAR Ion Propulsion System on the Deep Space One Mission," IEPC-01-075, October 2001.

${ }^{3}$ Soulas, G.C., Haag, T.W., and Patterson, M.J., "Performance Evaluation of $40 \mathrm{~cm}$ Ion Optics for the NEXT Ion Engine," AIAA 2002-3834, July 2002.

${ }^{4}$ Birdsall, C.K. and Langdon, A.B., Plasma Physics Via Computer Simulation, Adam Hilger Press, 1991.

${ }^{5}$ Bird, G.A., Molecular Gas Dynamics and the Direct Simulation of Gas Flows, Oxford University Press, 1994.

${ }^{6}$ G. Soulas, H. Kamhawi, M. Patterson, and M. Gardner, “NEXT Ion Engine 2000 h Wear Test Results,” AIAA 2004-3791, July 2004.

${ }^{7}$ H. Kamhawi, G. Soulas, M. Patterson, and M. Gardner, "NEXT Ion Engine 2000 Hour Wear Test Plume and Erosion Results," AIAA 2004-3792, July 2004.

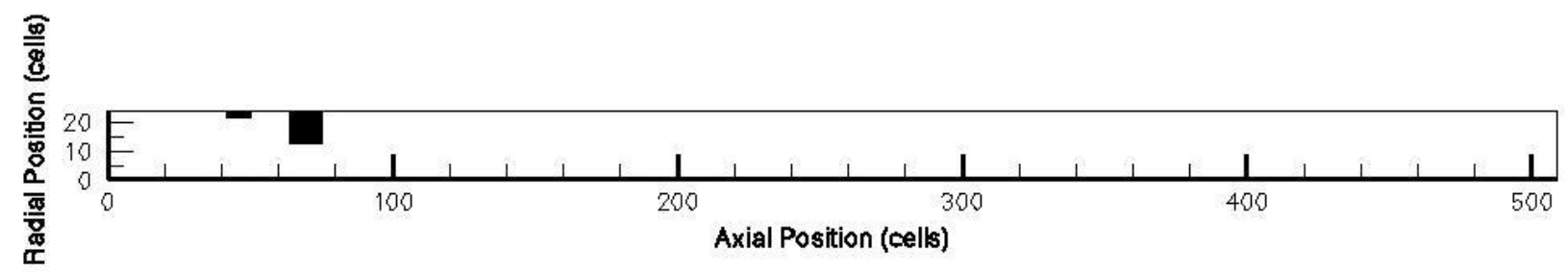

Figure 1. A typical computational domain. The axis labels represent the number of cells in each direction, and the black areas represent the ion optics. 


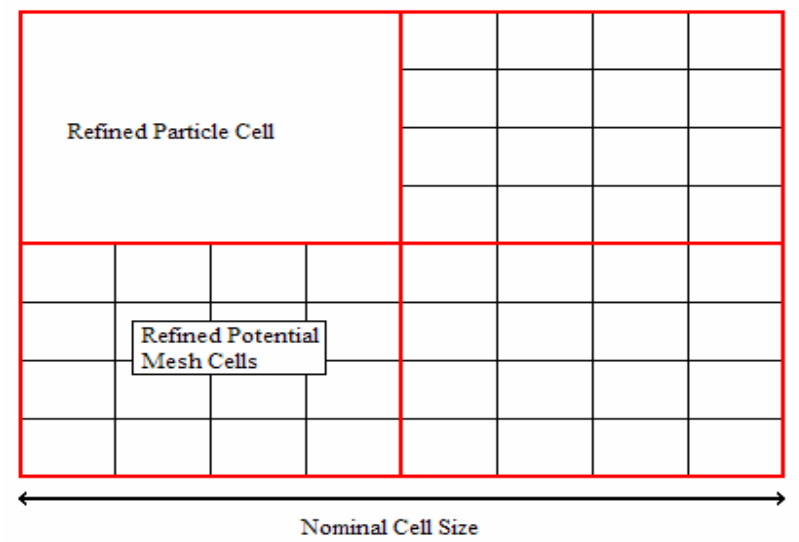

Figure 2. Demonstration of the meshes used in the model. The red grid indicates particle cells, while the black grid represents the potential mesh.

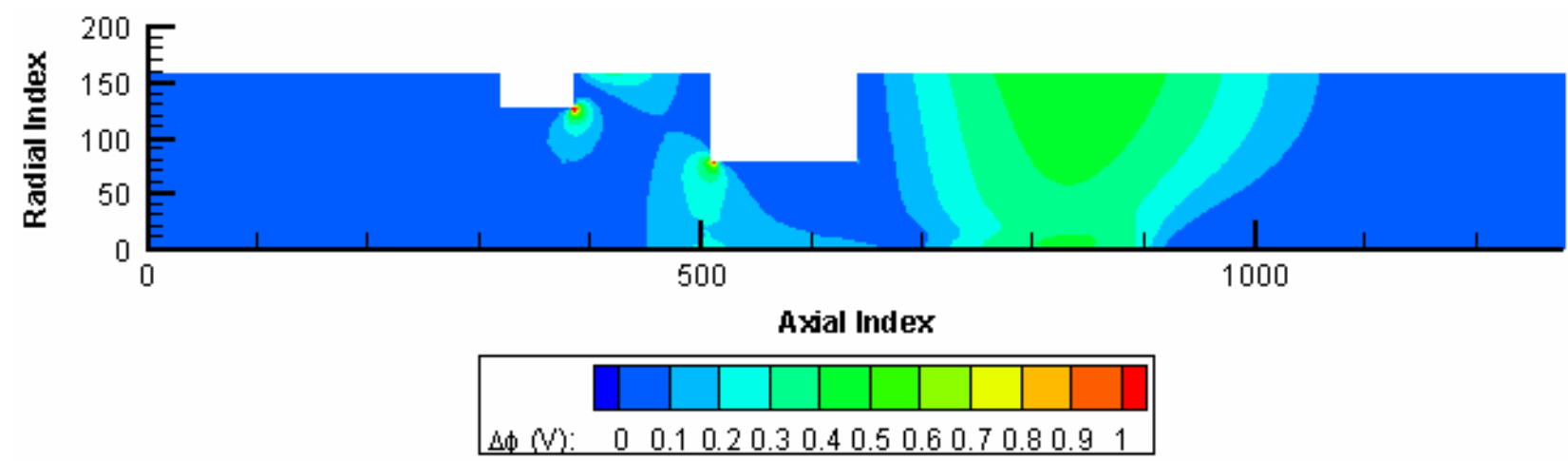

Figure 3. The difference in potential between an $8 x$ refined case and a 16x refined case. The difference near the optics is due to mesh coarseness error, and the difference downstream of the optics is due to residual error.

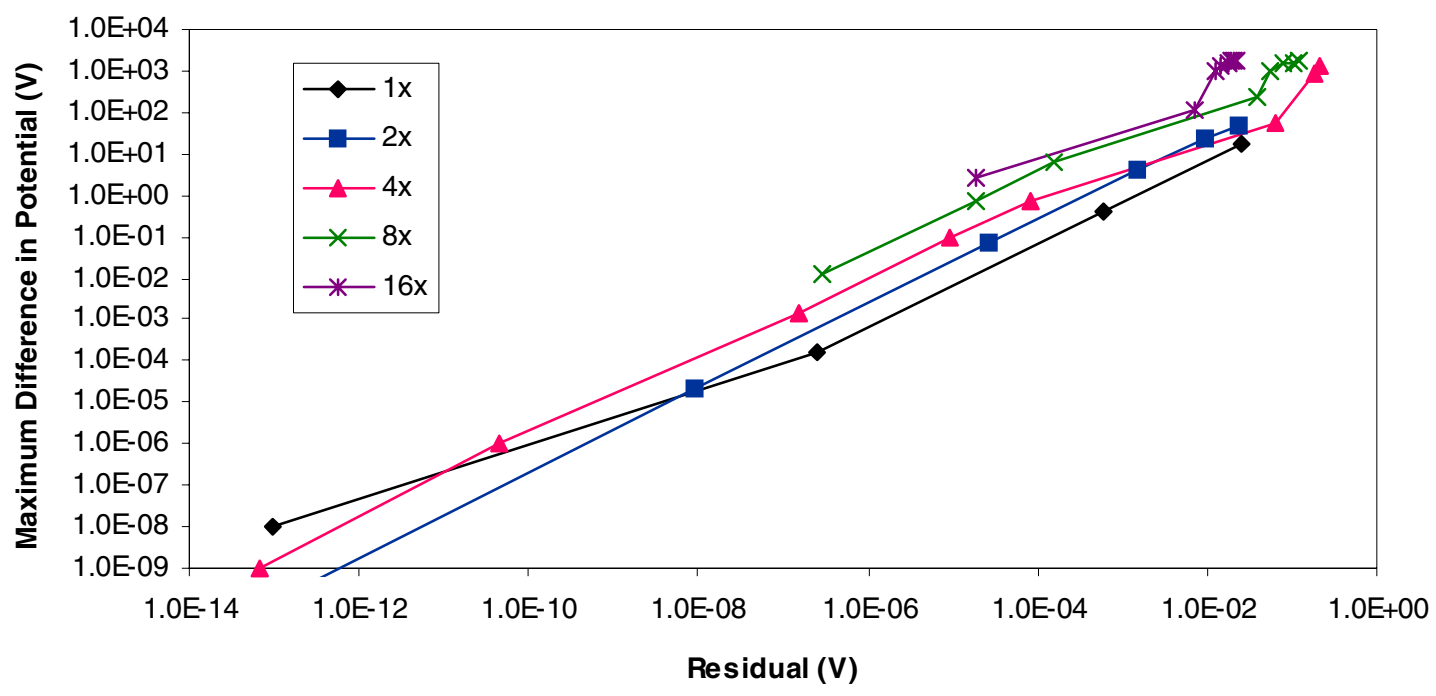

Figure 4. Maximum difference in potential as a function of residual. Higher levels of refinement require a lower residual in order to reach the same level of error. 


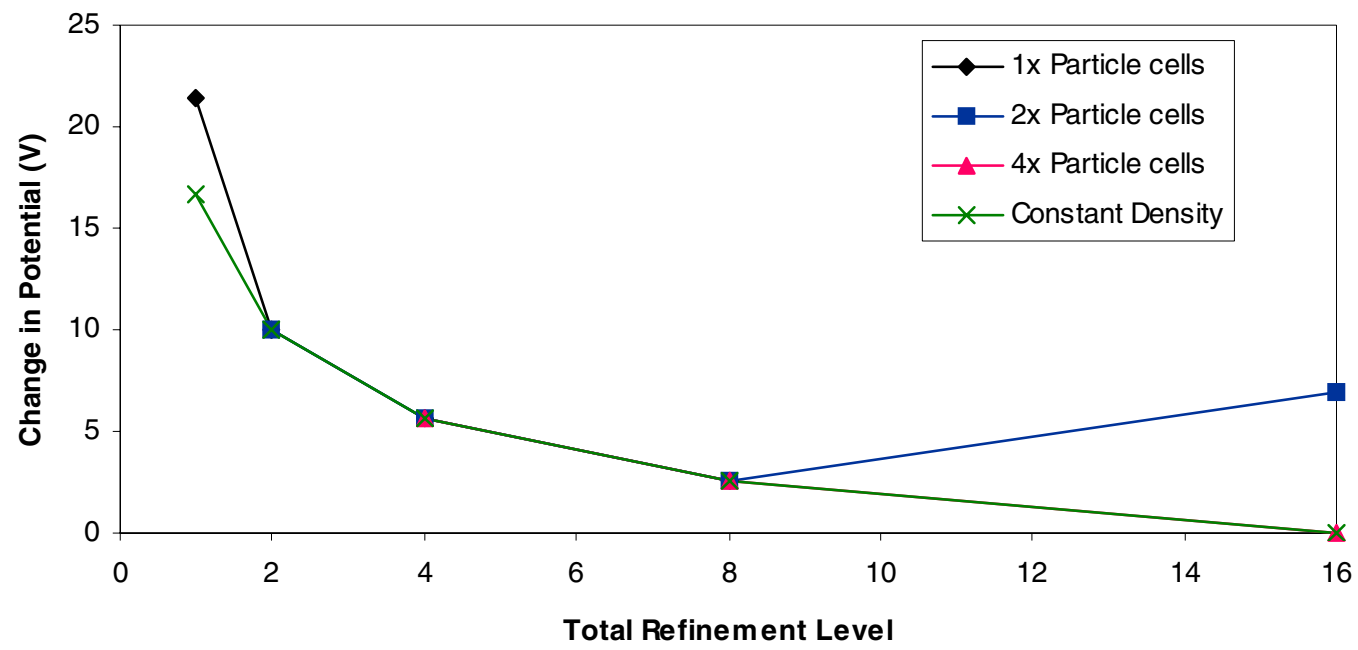

Figure 5. Maximum difference in potential as the mesh is refined. The anomalous point at 16x total refinement and $2 x$ particle cells is due to a high residual error.

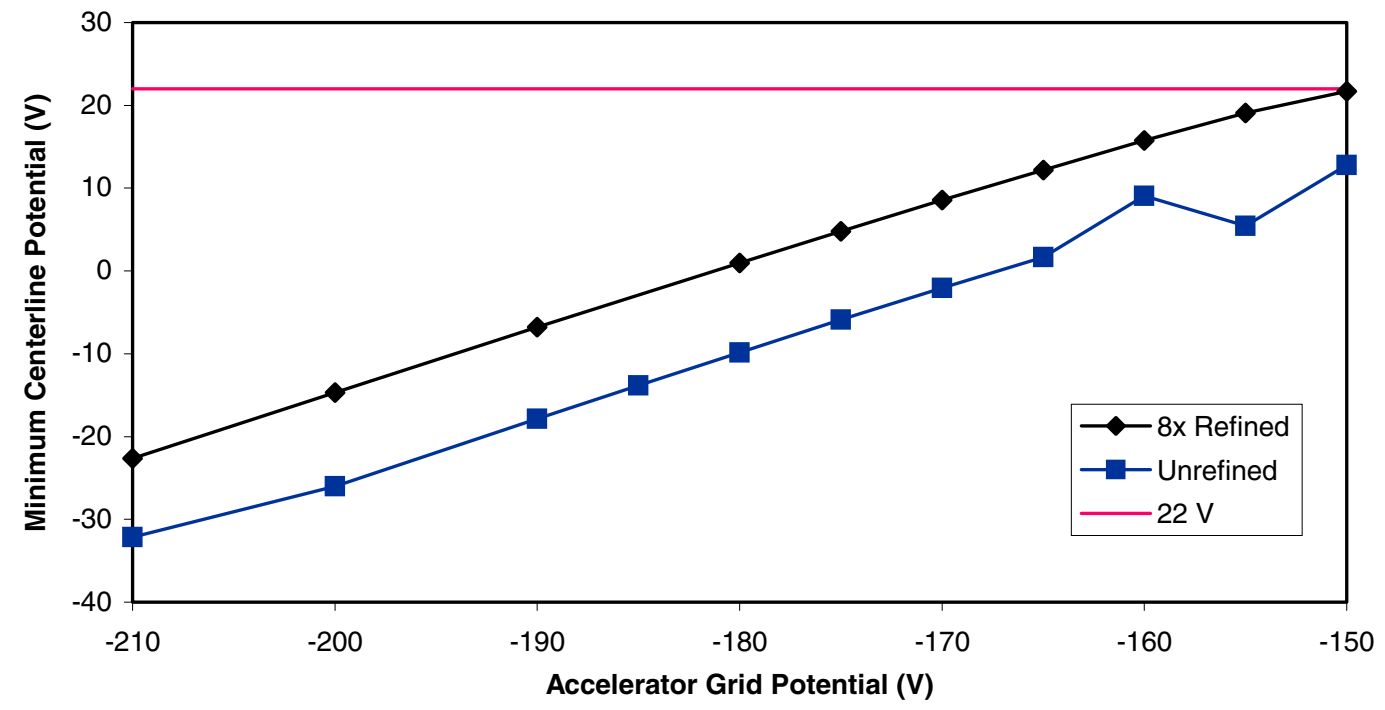

Figure 6. Minimum centerline potential as a function of accelerator grid potential. Electron backstreaming will occur when the minimum centerline potential reaches $22 \mathrm{~V}$. 


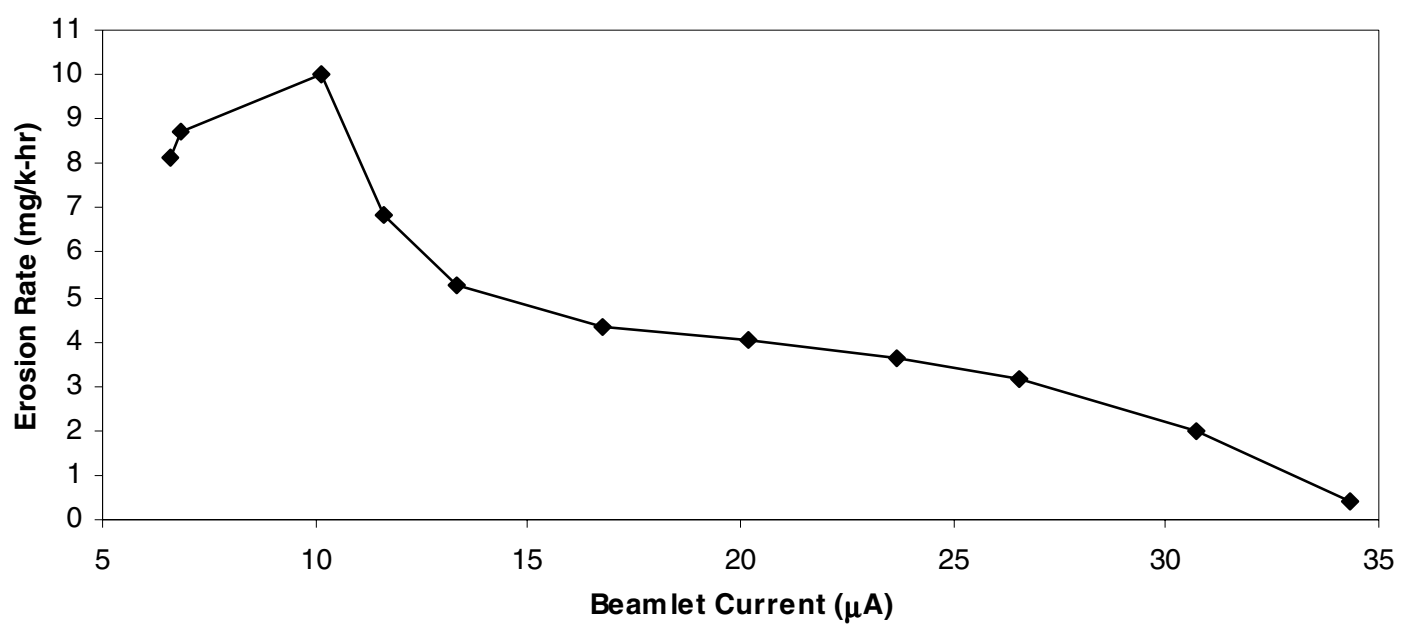

Figure 7. Erosion rate due to direct impingement of beam ions as a function of beamlet current.

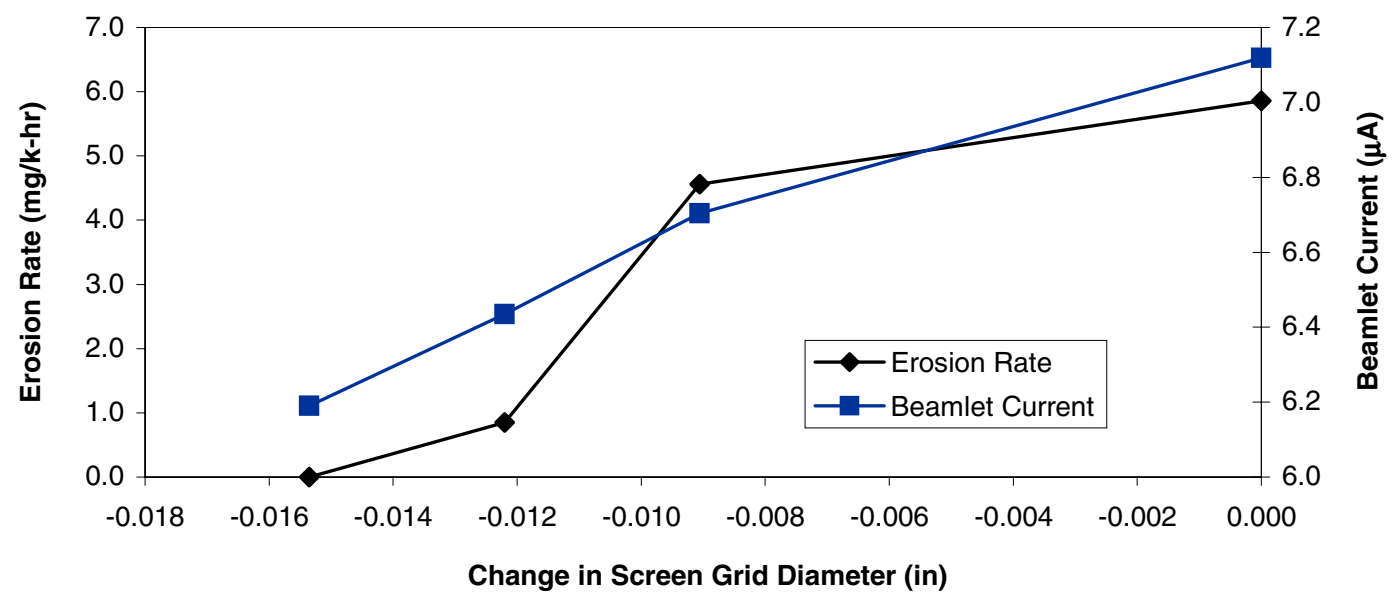

Figure 8. Change in erosion rate and beamlet current as the screen grid aperture diameter is decreased from the nominal size. 


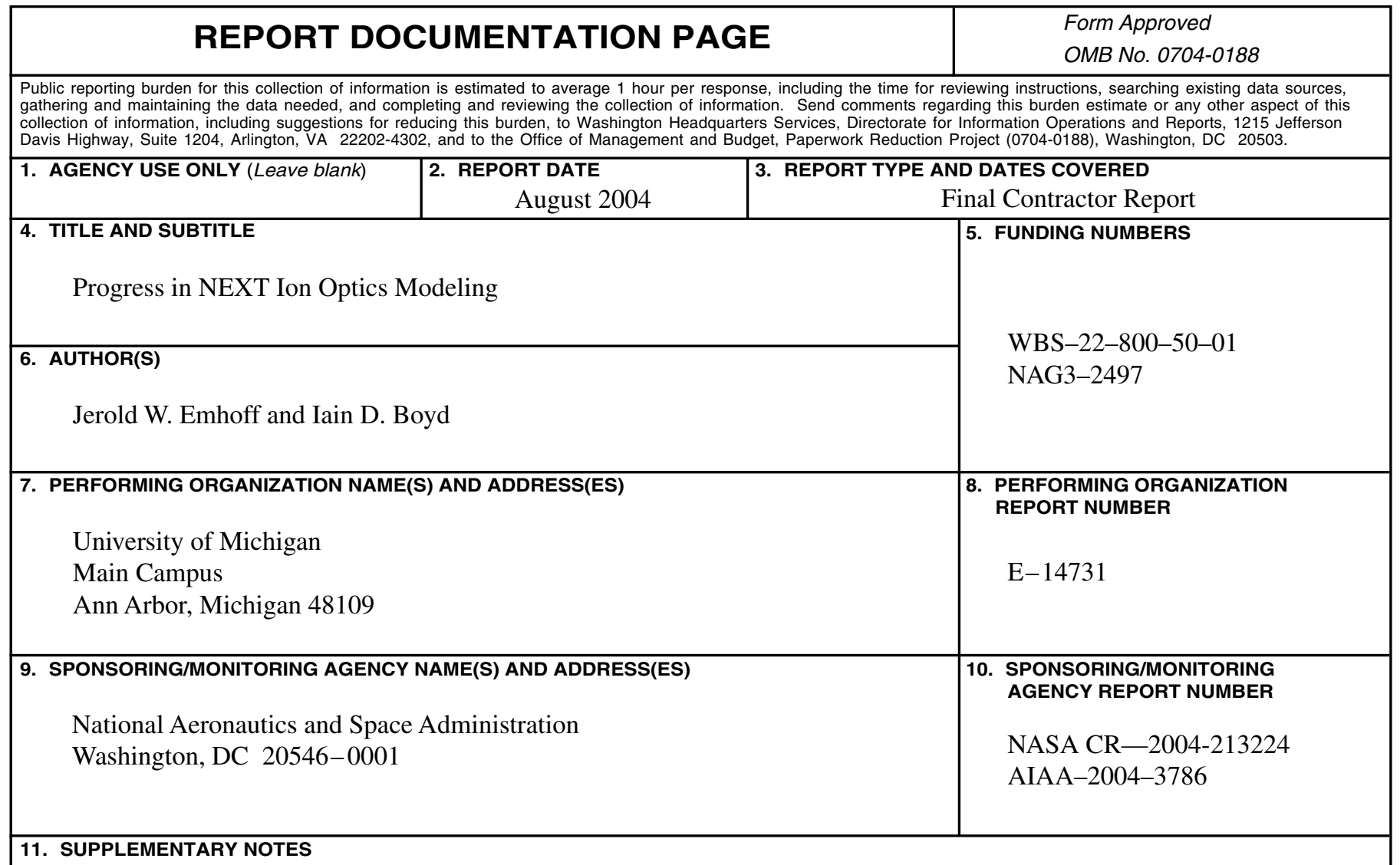

Prepared for the 40th Joint Propulsion Conference and Exhibit cosponsored by the AIAA, ASME, SAE, and ASEE, Fort Lauderdale, Florida, July 11-14, 2004. Project Manager, George C. Soulas, Power and On-Board Propulsion Technology Division, NASA Glenn Research Center, organization code 5430, 216-977-7419.

12a. DISTRIBUTION/AVAILABILITY STATEMENT

12b. DISTRIBUTION CODE

Unclassified - Unlimited

Subject Category: 20

Distribution: Nonstandard

Available electronically at http://gltrs.grc.nasa.gov

This publication is available from the NASA Center for AeroSpace Information, 301-621-0390.

13. ABSTRACT (Maximum 200 words)

Results are presented from an ion optics simulation code applied to the NEXT ion thruster geometry. The error in the potential field solver of the code is characterized, and methods and requirements for reducing this error are given. Results from a study on electron backstreaming using the improved field solver are given and shown to compare much better to experimental results than previous studies. Results are also presented on a study of the beamlet behavior in the outer radial apertures of the NEXT thruster. The low beamlet currents in this region allow over-focusing of the beam, causing direct impingement of ions on the accelerator grid aperture wall. Different possibilities for reducing this direct impingement are analyzed, with the conclusion that, of the methods studied, decreasing the screen grid aperture diameter eliminates direct impingement most effectively.

\section{SUBJECT TERMS}

Ion thruster; Ion engine; Ion propulsion

\begin{tabular}{|c|c|c|c|}
\hline $\begin{array}{c}\text { 17. SECURITY CLASSIFICATION } \\
\text { OF REPORT } \\
\text { Unclassified }\end{array}$ & $\begin{array}{c}\text { 18. SECURITY CLASSIFICATION } \\
\text { OF THIS PAGE } \\
\text { Unclassified }\end{array}$ & $\begin{array}{c}\text { 19. SECURITY CLASSIFICATION } \\
\text { OF ABSTRACT } \\
\text { Unclassified }\end{array}$ \\
\hline
\end{tabular}

NSN 7540-01-280-5500 

